\title{
The Fine Structure of Herman Rings
}

\author{
Núria Fagella* \\ Dept. de Matemàtiques i Informàtica \\ Barcelona Graduate School of Mathematics (BGSMath) \\ Universitat de Barcelona (UB) \\ Gran Via 585 \\ 08007 Barcelona, Spain \\ fagella@maia.ub.es \\ Christian Henriksen ${ }^{\dagger}$ \\ Dept. of Appl. Math. and Comp. Science \\ Technical University of Denmark \\ Matematiktorvet \\ Building 303 B, room 154 \\ 2800 Kgs. Lyngby, Denmark \\ chrh@dtu.dk
}

March 28, 2017

\begin{abstract}
We study the geometric structure of the boundary of Herman rings in a model family of Blaschke products of degree 3 (up to quasiconformal deformation). Shishikura's quasiconformal surgery relates the Herman ring to the Siegel disk of a quadratic polynomial. By studying the regularity properties of the maps involved, we transfer McMullen's results on the fine local geometry of Siegel disks to the Herman ring setting.
\end{abstract}

\section{Introduction}

We consider the dynamical system induced by the iterates of a rational map $f: \widehat{\mathbb{C}} \rightarrow \widehat{\mathbb{C}}$ of degree $d \geq 2$, where $\widehat{\mathbb{C}}$ denotes the Riemann sphere or compactified complex plane. We use the notation $f^{n}:=\overbrace{f \circ \cdots \circ f}^{n}$ to denote the $n^{\text {th }}$ iterate of $f$. Under this dynamics, the Riemann sphere splits into two completely invariant sets: the Fatou set, formed by those points for which the sequence $\left\{f^{n}\right\}$ is normal in some neighborhood, and its complement, the Julia set which is always non-empty. By definition the Fatou set is open and therefore the Julia set is a compact set of the sphere. Connected components of the Fatou set, also known as the Fatou components, map onto one another and are eventually periodic [Sul85]. In contrast to this, the dynamics is chaotic on the Julia set. For background on the dynamics of rational maps we refer for example to [CG93] and [Mil06].

*Partially supported by spanish grants MTM2011-26995-C02-02, MTM2014-52209-C2-2-P and MDM-20140445 María de Maeztu.

${ }^{\dagger}$ Partially supported by the Danish Council for Independent Research grant DFF-4181-00502

2000 Mathematics Subject Classification: Primary 37F10. Secondary 30D20. 
An especially relevant particular case of rational maps are polynomials, which are exactly (up to Möbius conjugation) those rational maps for which infinity is a fixed point and has no preimages other than itself. In particular this implies that infinity is a superattracting fixed point, and the dynamics is locally conjugate to $z \mapsto z^{d}$ around this point for some $d \geq 2$, the degree of the polynomial; it also means that the basin of attraction of infinity, that is the set of points attracted to infinity under iteration, is connected and completely invariant. The boundary of this basin is compact in $\mathbb{C}$ and coincides with the Julia set of the polynomial.

Periodic Fatou components of rational maps $f$ are completely classified [Fat19]: a periodic component $U$ is either part of a basin of attraction of an attracting or parabolic cycle, or a rotation domain, which means that some iterate of $\left.f\right|_{U}$ is conformally conjugate to a rigid rotation by an irrational multiple of $2 \pi$, called the rotation number. Rotation domains may be simply connected in which case they are called Siegel disks, or doubly connected in which case they are known as Herman rings. By definition, Herman rings separate the Julia set and have a disconnected boundary.

The dynamics of a rational map is determined, to a large extent, by the orbits of its critical points, i.e. the zeros of its derivative. Indeed, any basin of attraction must contain a critical point [Fat19] and every boundary component of a rotation domain is accumulated by a critical orbit (i.e. the orbit of a critical point) [Fat19, Shi87]. In some cases the relation is even stronger: any rotation domain with rotation number of bounded type (see Section 1.1) have Jordan boundaries which actually contain some critical point [Zha11]. In this work we will only consider rotation domains with this property.

Herman rings are undoubtedly the least well known among all possible types of periodic Fatou components of rational maps, one reason being that they are not associated to any periodic point with a certain multiplier, as the other types (basins of attraction or Siegel disks) are. Their closest relatives, Siegel disks, are much better understood, both in terms of conditions for their existence and in terms of the different properties that their boundaries possess. Relevant to our work will be, for instance, the string of geometric results about the fine structure of Siegel disks, proven by McMullen in [McM98], such as self-similarity of the Siegel disk around the critical point or measurable depth of the critical point in the filled Julia set (see Section 2).

But there is a procedure to relate Siegel disks and Herman rings, known as Shishikura's surgery (see Section 3 and [Shi87]). Roughly speaking, starting with a map that has a Herman ring $H$ (of a certain rotation number), this construction produces a map having a Siegel disk $S$ (of the same rotation number); at the same time it relates both functions via a quasiconformal map $\Phi$ which is a partial conjugacy between them. Intuitively, Shishikura's procedure erases the hole from the Herman ring (and from all its preimages), substituting the dynamics there by a rigid rotation (see Figure 1). The procedure is reversible, and it therefore ties certain problems on Herman rings (such as their existence for given rotation numbers) to the corresponding problem for Siegel disks.

The quasiconformal map $\Phi$ mentioned above opens up a possibility to transfer geometric properties between Siegel disks and their corresponding Herman rings. Some of them are fairly obvious to transport: If the boundary of $S$ is a Jordan curve, so will be both boundary components of $H$; or they will all contain a critical point or none will. But other geometric properties are not necessarily preserved by general homeomorphisms or quasiconformal maps.

In this paper we study the extra regularity properties of the quasiconformal map $\Phi$ and use them to transfer some of McMullen's results about the fine geometry of Siegel disks to corresponding statements about Herman rings. These are the principal contents of Theorems A and B. In the latter, we additionally conclude that the full boundary of the Herman ring is, surprisingly, tightly similar to that of a Siegel disk, even though one of them is disconnected and the other is not. The concept of tight similarity, introduced here, is stronger than regular similarity. In other words, zooming in around the critical 
point, the holes of the Herman ring tend to become invisible, until the Siegel disk and the Herman ring become indistinguishable form each other (see Figure 2).

Our study is done using a model family of rational maps of degree 3, which is one of the simplest that exhibits Herman rings of all rotation numbers of Brjuno type (see section 1.1). It also has the property that Shishikura's surgery relates it to the family of quadratic polynomials used in McMullen's results. However, in the same way that McMullen's properties also hold for quadratic-like mappings (i.e., degree 2 holomorphic branched coverings which map a topological disk properly over itself, see [DH85]), our theorems also extend to appropriate rational-like maps (see Remark 1.5).

\section{Acknowledgements}

The authors are grateful to Carsten Lunde Petersen and Saeed Zakeri for their insightful comments. They also thank the Institut de Matemtica at Universitat de Barcelona and the Department of Applied Mathematics and Computer Science of the Technical University of Denmark for their hospitality while this work was in progress. Final thanks go to the referee for many detailed suggestions that made the paper better.

\subsection{Setup and statement of results}

Arithmetics plays an important role in the dynamics of rotation domains. It is important to distinguish between three nested classes of irrational numbers. For $a_{1}, a_{2}, \ldots \in \mathbb{N}$, we let

$$
\left[a_{1}, a_{2}, \ldots\right]=\frac{1}{a_{1}+\frac{1}{a_{2}+\frac{1}{\ddots}}}
$$

denote the continued fraction expansion with $a_{1}, a_{2}, \ldots$ as coefficients, and denote the convergents by

$$
\frac{p_{n}}{q_{n}}=\left[a_{1}, a_{2}, \ldots, a_{n}\right] .
$$

See [Khi97] for details. An irrational number $\theta$ is a quadratic irrational if the sequence of coefficents $a_{1}, a_{2}, \ldots$ is eventually periodic. The quadratic irrationals are exactly the irrational roots of quadratic equations with integer coefficients.

A more general set of irrational numbers are the those of bounded type. They are numbers whose coefficients satisfy $\sup a_{n}<\infty$.

An even more general class of irrationals is the class of Brjuno numbers which we denote by $\mathcal{B}$. A Brjuno number is charaterized by the denominators of its convergents; a number is Brjuno if and only if $\sum \log q_{n+1} / q_{n}<\infty$.

These classes of irrational numbers are relevant to dynamics. If $f$ is a holomorphic map in a neighborhood of the origin, such that $f(0)=0$ and $f^{\prime}(0)=e^{2 i \pi \alpha}$ with $\alpha \in \mathcal{B}$, then there is a neighborhood of 0 on which $f$ is conjugate to the rigid rotation $z \mapsto e^{2 \pi i \alpha} z$ [Sie42, Brj65, Rüs67]. If the map $f$ is globally defined, this neighborhood is part of a Siegel disk. Conversely, if a quadratic polynomial has an invariant Siegel disk, its center is a fixed point with multiplier $e^{2 i \pi \alpha}$ with $\alpha \in \mathcal{B}$ [Yoc95].

Here and in the rest of the article, we fix an irrational number $\theta$ of bounded type. We let $\lambda=e^{2 i \pi \theta}$, and fix the quadratic polynomial

$$
P(z)=\lambda z+z^{2} .
$$

This polynomial has a unique critical point $\omega:=-\frac{\lambda}{2}$. The origin is a fixed point of multiplier $P^{\prime}(0)=\lambda$. We know that $P$ posseses a Siegel disk $S$ centered at $z=0$, because the numbers of bounded type form a subset of the Brjuno numbers. 
We will work with the simplest family of rational maps that can possess Herman rings, namely

$$
f_{a, b}(z):=b z^{2} \frac{a z+1}{z+a}
$$

for $a, b \in \mathbb{C}$ and $b \neq 0$. Every $f_{a, b}$ has superattracting fixed points at the origin and at infinity. Additionally, there are two other criticial points which we denote by $\omega_{1}$ and $\omega_{2}$.

It is well known [Brj65, Shi87, Yoc95] that there exists $a, b$ such that $f_{a, b}$ has a Herman ring with rotation number $\alpha$ if and only if $\alpha$ is Brjuno. In [BFGH05], Buff, Fagella, Geyer and Henriksen show that for every Brjuno number $\alpha$, there exists a punctured disk holomorphically embedded in the $a, b$ parameter space of $f_{a, b}$, such that every mapping in the disk possesses an invariant Herman ring with rotation number $\alpha$. Note that $f_{a, b}$ is not a Blaschke product unless $a$ is real. But every map $f_{a, b}$ with a Herman ring is quasiconformally conjugate to a Blaschke product (for which $a \in \mathbb{R}$ and $|b|=1$ ), and the conjugacy can be chosen to be conformal off a small neighborhood of the core curve of the Herman ring and its preimages.

From now on, we let $a, b$ be parameters chosen such that $f_{a, b}$ has an invariant Herman ring with rotation number $\theta$. Since we shall not vary $a, b$, we simply denote the this map by $f$. Hence

$$
f(z)=b z^{2} \frac{a z+1}{z+a}
$$

has a Herman ring $H$ of rotation number $\theta$. The Herman ring has two boundary components $\partial_{j} H, j=1,2$, which are both quasicircles, each containing a critical point of $f$ (this follows from results of Herman, Ghys, Douady, Światek and Shishikura, see e.g. [BF14, Sections 7.2 and 7.3]). We label the components such that $\partial_{1} H$ is contained in the bounded component of the complement of $H$, and we label the critical points such that $\omega_{j} \in \partial_{j} H, j=1,2$.

Let $A_{f}(\infty)$ denote the immediate basin of attraction of the fixed point at infinity. The boundary of $A_{f}(\infty)$ is a proper subset of the Julia set $J(f)$. This is in contrast to what happens for $P$, the quadratic polynomial in (2), where the boundary of the basin of infinity, $A_{P}(\infty)$, coincides with $J(P)$.

In [Shi86], Shishikura introduced a surgery that could turn a map with a cycle of Herman rings into a map with a cycle and Siegel disk and vice versa (see Section 3). We will use a special case of his construction in the following theorem.

Theorem 1.1. Let $\theta$ be of bounded type and let $P$ and $f$ be as in (2) and (3). In the setup above, there exists a P-invariant simply connected domain $D$ compactly contained in $S$, and a quasiconformal mapping $\Phi: \widehat{\mathbb{C}} \rightarrow \widehat{\mathbb{C}}$, such that

(a) $\Phi \circ P=f \circ \Phi$ on $\widehat{\mathbb{C}} \backslash D$

(b) $\Phi$ maps $J(P)$ onto $\partial A_{f}(\infty)$, $\partial S$ onto $\partial_{2} H$, and $\omega$ to $\omega_{2}$.

(c) $\bar{\partial} \Phi=0$ a.e. outside $\mathcal{D}:=\bigcup_{n=0}^{\infty} P^{-n}(D)$.

(d) $\Phi$ is $C^{1+\alpha}$-conformal at $\omega$ with $\Phi^{\prime}(\omega) \neq 0$.

The notion of $C^{1+\alpha}$-conformality, due to McMullen, is defined as follows.

Definition $1.2\left(C^{1+\alpha}\right.$ conformality). A mapping $\phi$ is $C^{1+\alpha}$-conformal at $z_{0}$ if there exist $\alpha, \delta, M>0$ such that

$$
\phi(z)=\phi\left(z_{0}\right)+\phi^{\prime}\left(z_{0}\right)\left(z-z_{0}\right)+R(z)
$$

holds with

$$
|R(z)| \leq M\left|z-z_{0}\right|^{1+\alpha}
$$

whenever $\left|z-z_{0}\right|<\delta$.

Note that this is stronger than $\mathbb{C}$-differentiability at the point $z_{0}$. We say $\phi$ is $C^{1+\alpha_{-}}$ anticonformal at $z_{0}$ if $\bar{\phi}$ is $C^{1+\alpha}$-conformal at $z_{0}$. 

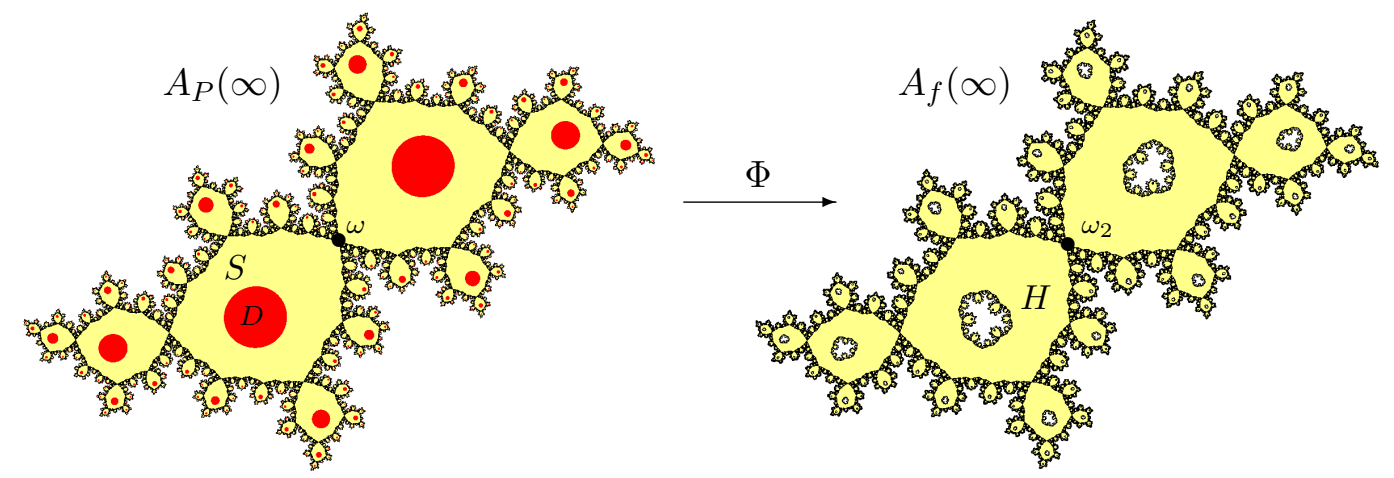

Figure 1: Illustration of Theorem 1.1 for $\theta=\frac{\sqrt{5}-1}{2}$, the golden mean. The dynamics of $P$ is shown on the left, which is symmetric with respect to $\omega$. We have colored the completely invariant set $\mathcal{D}$ in red, and $J(P)$ in black. The dynamics of $f$ is shown on the right, which has been rotated, scaled and translated to illustrate the similarity with $P$. The Herman ring and its preimages are colored yellow and $J(f)$ black. By Theorem 1.1, there exists a quasiconformal homeomorphism $\Phi$ which is conformal outside the red set. Outside $D, \Phi$ conjugates $P$ to $f$, and $\Phi$ is $C^{1+\alpha}$-conformal at $\omega$.

Theorem 1.1 is illustrated in Figure 1. The fact that the quasiconformal conjugacy provided by Theorem 1.1 is $C^{1+\alpha}$ at $\omega$ implies that the boundary components of $H$ are locally similar to the boundary of $S$ near respective critical points. To make a precise statement, we introduce a notion of similarity that is stronger than Tan Lei's notion of asymptotic similarity introduced in [Tan90]. Let $B(c, r)$ denote the open ball of center $c \in \mathbb{C}$ and radius $r>0$.

Definition 1.3 (Tight similarity). We say that two compact sets $A, B$ are tightly similar at $z_{0}$ if there exist $\delta, \beta>0$ and $L>0$ such that

(1) $a \in A \cap B\left(z_{0}, \delta\right) \Rightarrow d(a, B) \leq L\left|a-z_{0}\right|^{1+\beta}$

(2) $b \in B \cap B\left(z_{0}, \delta\right) \Rightarrow d(b, A) \leq L\left|b-z_{0}\right|^{1+\beta}$

When $A$ and $B$ are tightly similar at $z_{0}$, we write

$$
A \underset{z_{0}}{\sim} B
$$

A compact set $A$ is tightly self-similar around $z_{0} \in A$ if

$$
A-z_{0} \underset{0}{\sim} \kappa\left(A-z_{0}\right)
$$

for some $\kappa \in \mathbb{C}$, with $|\kappa|>1$.

It is easy to check that tight similarity at $z_{0}$ is an equivalence relation on the compact subsets of $\mathbb{C}$. The relation between tight similarity and Hausdorff distance $d_{H}$ between compact sets is as follows. If $A$ and $B$ are two compact sets and we set $A_{r}=(A \cap$ 
$\left.\overline{B\left(z_{0}, r\right)}\right) \cup \partial B\left(z_{0}, r\right)$ and $B_{r}=\left(B \cap \overline{B\left(z_{0}, r\right)}\right) \cup \partial B\left(z_{0}, r\right)$, then tight similarity at $z_{0}$ is equivalent to

$$
d_{H}\left(A_{r}, B_{r}\right)=\mathcal{O}\left(r^{\lambda}\right),
$$

for some $\lambda>1$ as $r \rightarrow 0$.

Main Theorem. Let $\theta$ be of bounded type and let $P$ and $f$ be as in (2) and (3). In the setup above, the following are satisfied.

(a) There exists a scaling factor $L \in \mathbb{C} \backslash\{0\}$ such that

$$
L(J(P)-\omega) \underset{0}{\widetilde{\partial}} \partial A_{f}(\infty)-\omega_{2} \underset{0}{\widetilde{0}} J(f)-\omega_{2} .
$$

(b) When $\theta$ is a quadratic irrational, $\partial_{2} H$ is tightly self-similar around $\omega_{2}$.

(c) The Siegel disk $S$ contains a Euclidean triangle with vertex at $\omega$ if and only if $H$ contains a Euclidean triangle with vertex at $\omega_{2}$.

The Main Theorem is illustrated in Figure 2, where we can see the similarity between $J(P), \partial A_{f}(\infty)$ and $J(f)$. Even though $J(f)$ and $\partial A_{f}(\infty)$ are topologically very different, as we blow up a neighborhood of $\omega_{2}$, the "holes" in the preimages of the Herman ring shrink faster than the blow-up rate.

In the illustration, it seems that the holes in the preimages of the Herman ring get increasingly small relative to the size of the preimage and not only relative to the rate of blow-up. Also, it seems that the holes are located further away from the "roots" of the preimages as we blow up. These experimental observations do not follow from the Main Theorem, and we do not know if they are true though we suspect that this behavior does occur quite generally.

The Main Theorem is useful in the numerical search for mappings in the family $f_{a, b}$ with Herman rings of bounded type rotation number. Indeed, if $q_{n}$ denotes the denominator of the convergents to $\theta$ defined in (1), from Theorem 1.1 we know

$$
\begin{aligned}
\frac{f_{a, b}^{q_{n+1}}\left(\omega_{j}\right)-\omega_{j}}{f_{a, b}^{q_{n}}\left(\omega_{j}\right)-\omega_{j}} & =\frac{\Phi\left(P^{q_{n+1}}(\omega)\right)-\Phi(\omega)}{\Phi\left(P^{q_{n}}(\omega)\right)-\Phi(\omega)} \\
& =\frac{P^{q_{n+1}}(\omega)-\omega+\mathcal{O}\left(\left|P^{q_{n+1}}(\omega)-\omega\right|^{1+\alpha}\right)}{P^{q_{n}}(\omega)-\omega+\mathcal{O}\left(\left|P^{q_{n}}(\omega)-\omega\right|^{1+\alpha}\right)} \\
& =\frac{P^{q_{n+1}}(\omega)-\omega}{P^{q_{n}}(\omega)-\omega}\left(1+\mathcal{O}\left(\left|P^{q_{n}}(\omega)-\omega\right|^{1+\alpha}\right)\right)
\end{aligned}
$$

for $j=1,2$. Therefore the points $(a, b)$ where $f_{a, b}$ has a Herman ring with rotation number $\theta$ satisfy

$$
\frac{P^{q_{n+1}}(\omega)-\omega}{P^{q_{n}}(\omega)-\omega} \approx \frac{f_{a, b}^{q_{n+1}}\left(\omega_{j}\right)-\omega_{j}}{f_{a, b}^{q_{n}}\left(\omega_{j}\right)-\omega_{j}}, \quad j=1,2,
$$

when $n$ is large, which narrows the search to a one-complex-dimensional set of parameters.

Let us finally note that we can use the dynamics to extend the results to all iterated preimages of the critical points:

Corollary 1.4. Suppose that $\omega^{\prime}$ and $u$ satisfy $P^{n}\left(\omega^{\prime}\right)=\omega$ and $f^{m}(u) \in\left\{\omega_{1}, \omega_{2}\right\}$ for some integers $n, m>1$. Then,

(a) $\Phi$ is $C^{1+\alpha}$-conformal at $\omega^{\prime}$.

(b) There exists a scaling factor $L^{\prime} \in \mathbb{C} \backslash\{0\}$ such that

$$
L^{\prime}\left(J(P)-\omega^{\prime}\right) \underset{0}{\sim} J(f)-u .
$$




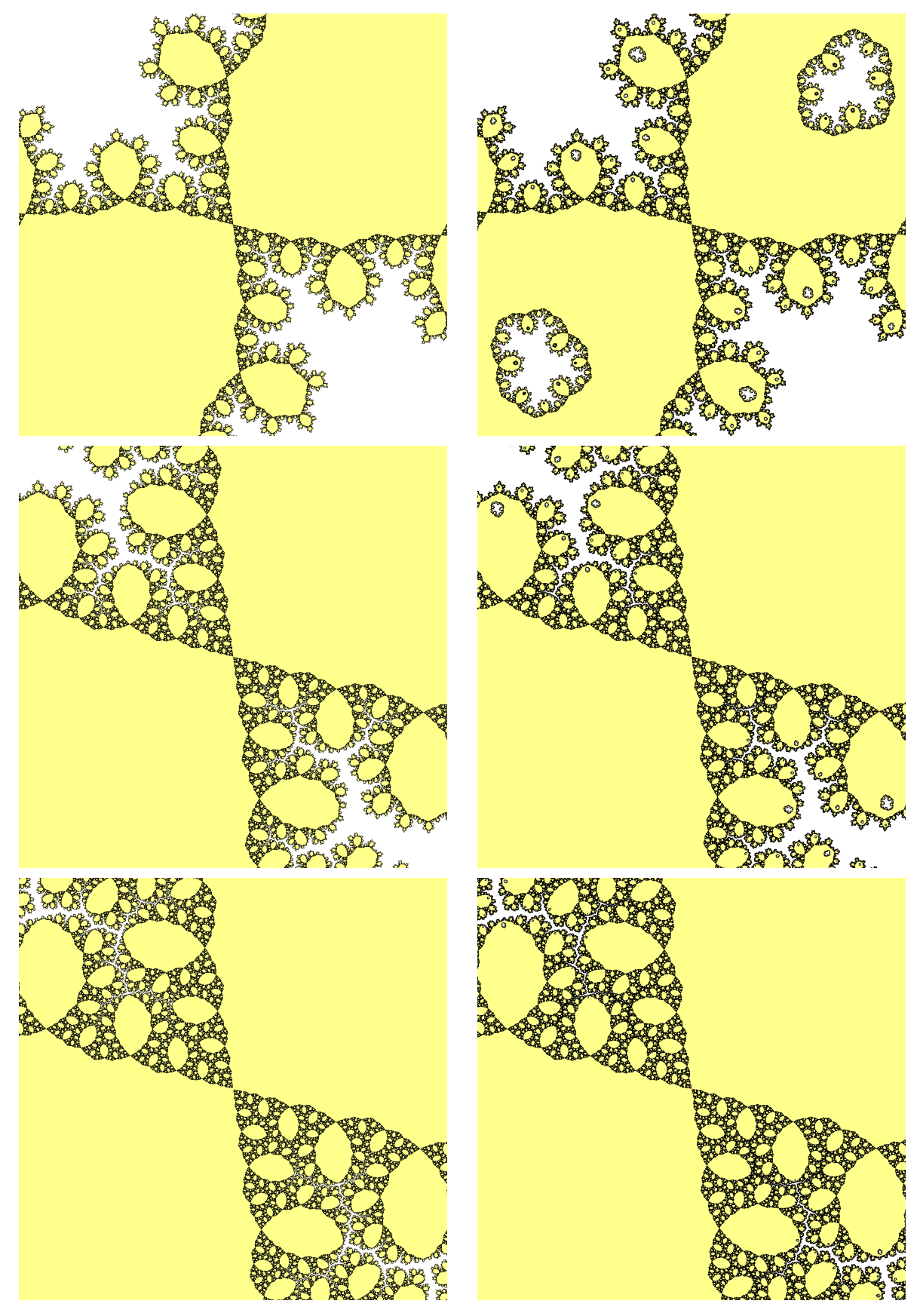

Figure 2: On the left column Julia sets of the quadratic polynomial $P$ as in (2) with $\theta=\frac{\sqrt{5}-1}{2}$ is shown. To the right is illustrated the Julia set of $f$ as in (3), with $(a, b)$ chosen so that $\theta$ is as above. Going down, we zoom in around $\omega$ and $\omega_{2}$ respectively, and see that similarity becomes more and more pronounced. 
(c) If $\theta$ is a quadratic irrational, $H^{\prime}$ is some connected component of $f^{-k}(H), k \geq 0$, and $u \in \partial H^{\prime}$, then $\partial H^{\prime}$ is tightly self-similar around $u$.

Remark 1.5. McMullen's results extend to a much more general class than quadratic polynomials, namely to all quadratic-like maps with a fixed point of derivative $e^{2 \pi i \theta}, \theta$ being irrational of bounded type (see [McM98, Theorem 5.1]). Using this result, one can see (not without some work) that Theorems A and B also extend to a more general setting than the model cubic family considered above. More precisely, if $g$ is a rational-like map that straightens to a member of the model family $f_{a, b}$ having a Herman ring of rotation number $\theta$, then our results apply to the "small Julia set" of $g$.

\section{Preliminaries about $P$ and its Siegel disk}

Before proving our two main theorems, we review some facts about the Julia set $J(P)$ and the Siegel disk $S$.

Recall that $P(z)=e^{2 i \pi \theta} z+z^{2}$, with $\theta$ of bounded type, and $\omega=-\frac{e^{2 \pi i \theta}}{2}$ is the critical point. In [Pet96], Petersen showed the following theorem.

Theorem 2.1. The Julia set $J(P)$ is locally connected and has zero Lebesgue measure.

McMullen proved a string of geometric results in [McM98]. He showed that $J(P)$ has Hausdorff dimension strictly less than two. He also showed that $\omega$ is a measurable deep point of a subset of the filled in Julia set $K(P):=\mathbb{C} \backslash A_{P}(\infty)$, a condition that is stronger than being a density point of $K(P)$.

Definition 2.2 (Measurable deep point). Let $z_{0} \in \mathbb{C}$ and $E$ be a Borel set. We call $z_{0}$ a measurable deep point of $E$ if there exist constants $M, \beta, \delta>0$ such that

$$
\operatorname{Area}\left(B\left(z_{0}, r\right) \backslash E\right) \leq M r^{2+\beta} \text {, whenever } r<\delta .
$$

Observe that if $E \subset E^{\prime}$ are Borel sets and $z_{0}$ is a measurable deep point of $E$ then $z_{0}$ is a measurable deep point of $E^{\prime}$.

Theorem 2.3 ([McM98, Cor. 4.5]). Let $\epsilon>0$ be arbitrary and define

$$
S_{\epsilon}=\left\{z \in K(P): d\left(P_{c}^{n}(z), S\right)<\epsilon \text { for all } n \geq 0\right\} .
$$

Then $\omega$ is a measurable deep point of $S_{\epsilon}$.

If $\phi: \widehat{\mathbb{C}} \rightarrow \widehat{\mathbb{C}}$ is quasiconformal and the support of the Beltrami coefficient $\mu_{\phi}:=\bar{\partial} \phi / \partial \phi$ gets thin close to a point $z_{0}$, we can expect $\phi$ to be regular at $z_{0}$. There are several results in this direction (see e.g. [LV73, Chapter 6]), and we will find use for the following theorem of McMullen.

Theorem 2.4 ([McM96, Theorem 2.19]). Suppose $\phi: U \rightarrow V$ is quasiconformal and let $\Omega=U \backslash \operatorname{supp} \mu_{\phi}$. If $z_{0}$ is a measurable deep point of $\Omega$, then $\phi$ is $C^{1+\alpha}$-conformal at $z_{0}$ for some $\alpha>0$, and $\phi^{\prime}\left(z_{0}\right) \neq 0$.

Remark 2.5. The last conclusion, the derivative being nonzero, is implicitly used in McMullen's text although not explicitly stated. For completeness, let us show how it follows from the condition of being measurable deep. In [LV73, Lemma 6.1] it is proven that if the dilatation $D_{\phi}(z)$ of $\phi$ at $z$, satisfies the convergence condition

$$
\iint_{|z|<r} \frac{D_{\phi}(z)-1}{|z|^{2}} d x d y<\infty
$$

for every small $r$, then $\phi$ is complex differentiable at 0 with nonzero derivative. By breaking the disk of radius $r$ into a union of annuli $A_{n}=\left\{\frac{1}{2^{n+1}}<|z|<\frac{1}{2^{n}}\right\}$, one can 
bound the integral on each annulus by $\frac{4(K-1) M}{2^{\beta n}}$, where $M$ and $\beta$ are the constants given by the condition of 0 being a measurable deep point of $\Omega$, and $K$ is the bound on the dilatation of $\phi$. The integral is thus bounded from above by a geometric series and hence finite.

McMullen also showed that when $\theta$ is a quadratic irrational, the Siegel disk is selfsimilar at the critical point $\omega$. More precisely he showed the following.

Theorem 2.6 ([McM98, Theorem7.1]). Suppose $\theta$ is a quadratic irrational, and let $s$ denote the eventual period of the coefficients of its continued fraction. Let $P$ be as in (2) and $S$ be its Siegel disk. Then, there exists a homeomorphism $\psi$ defined in some neighborhood of $\omega$ with $\psi(\omega)=\omega$, conjugating $P^{q_{n}}$ to $P^{q_{n+s}}$ on $\partial S$ for $n$ sufficiently large. Moreover,

$$
\psi(z)= \begin{cases}\omega+\kappa(z-\omega)+O\left((z-\omega)^{1+\alpha}\right) & \text { if s even } \\ \omega+\kappa(\overline{z-\omega})+O\left((z-\omega)^{1+\alpha}\right) & \text { if s odd }\end{cases}
$$

for some $\alpha>0$ and $\kappa \in \mathbb{C}$ with $0<|\kappa|<1$.

It follows from the theorem that when $s$ is even, $\psi$ is $C^{1+\alpha}$-conformal at $\omega$, and when $s$ is odd, $\psi$ is $C^{1+\alpha}$-anticonformal.

Remark 2.7 (Tight self-similarity of $S$ ). We will see later (see Remark 4.4) that this implies that the Siegel disk is actually tightly self-similar. More precisely, if $s$ is even, then

$$
\partial S-\omega \underset{0}{\sim} \kappa(\partial S-\omega),
$$

and, when $s$ is odd,

$$
\partial S-\omega \underset{0}{\sim} \kappa(\overline{\partial S-\omega}),
$$

where $\kappa$ is the scaling factor in Theorem 2.6.

Using the preceeding theorem, Buff and Henriksen [BH99] proved that for some values of $\theta$, such as the golden mean $\frac{\sqrt{5}-1}{2}$, the Siegel disk $S$ contains a Euclidean triangle with a vertex at the critical point.

\section{Quasiconformal surgery and Proof of Theorem 1.1}

In this section we prove Theorem 1.1. We shall see that parts (a), (b) and (c) follow directly from a surgery construction due to Shishikura, whereas part (d) can be derived by bounding the relative area of the support of the quasiconformal distorsion of $\Phi$ as we approach $\omega$. The main idea of the surgery is simply to replace the dynamics in the hole of the Herman ring with an irrational rotation. In this way we obtain a quasiregular map $F$ which is quasiconformally conjugate to $P$. Letting $\Phi$ denote the conjugacy from $P$ to $F$, we then check that it has the stated properties. Details are as follows (c.f. [Shi87] and [BF14, Section 7.2]).

We keep the notation from the setup in Section 1.1. Let $\phi_{0}: H \rightarrow\{z: r<|z|<1\}$ denote the linearizing map, conjugating $f$ to $R_{\theta}: z \mapsto e^{2 i \pi \theta} z$. Define three topological disks $U_{1} \Subset U_{2} \Subset U_{3}$, such that $\partial U_{1} \subset H$ and $\partial U_{2} \subset H$ are $f$-invariant curves and $U_{3}$ is the complement of the unbounded component of $\mathbb{C} \backslash H$. The image under $\phi_{0}$ of $\partial U_{1}$ is a circle, i.e., the boundary of a disk $V_{1}$. Similarly, we define $V_{2}$ to be the disk whose boundary is $\phi_{0}\left(\partial U_{2}\right)$, and we let $V_{3}=B(0,1)$. See Figure 3 .

We modify and extend $\phi_{0}$ to define it on all of $U_{3}$. Define $\phi_{1}: U_{3} \rightarrow V_{3}$ by letting it be equal to $\phi_{0}$ on $U_{3} \backslash U_{2}$, by requiring that it maps $\left(U_{1}, 0\right)$ conformally to $\left(V_{1}, 0\right)$ and interpolating quasiconformally on the annulus $U_{2} \backslash U_{1}$. This way we get a quasiconformal 

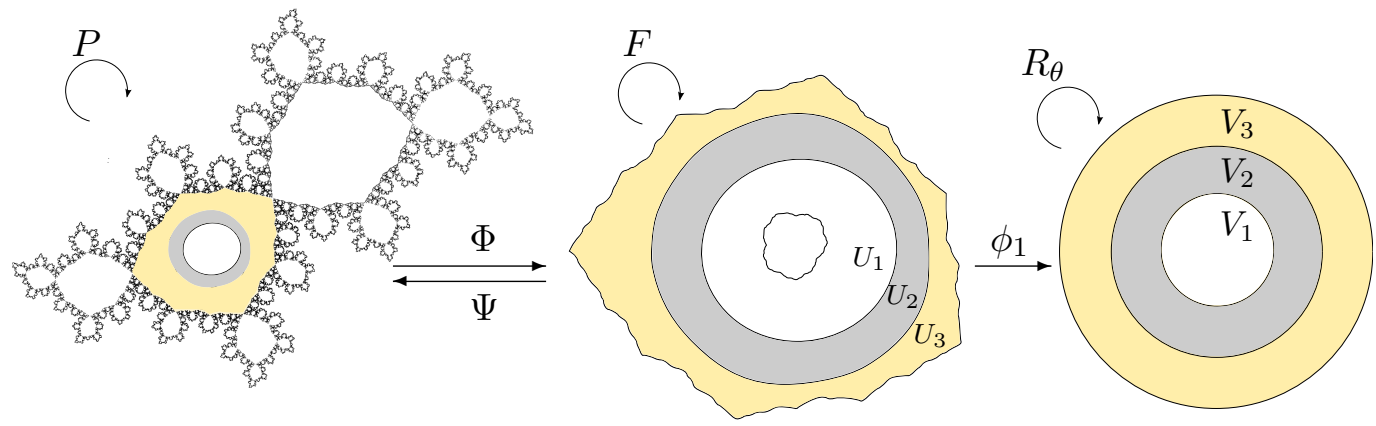

Figure 3: Quasiconformal surgery to produce the polynomial $P$ with a Siegel disk from the rational map $f$ with the Herman ring $H$.

mapping $\phi_{1}: U_{3} \rightarrow V_{3}$ which we shall use to paste the rigid rotation into the Herman ring.

Define

$$
F:= \begin{cases}f & \text { on } \widehat{\mathbb{C}} \backslash U_{2} \\ \phi_{1}^{-1} \circ R_{\theta} \circ \phi_{1} & \text { on } U_{2} .\end{cases}
$$

Note that the two maps agree on $\partial U_{2}$ so $F$ is continuous. This is a model of a quadratic polynomial, since the pole of $f$ no longer exists and the global degree is now two. It is, however, only quasiregular. To remedy this, we will define an $F$-invariant Beltrami coefficient $\mu$ with bounded dilatation with the intention of applying the Measurable Riemann Mapping Theorem. We start by defining $\mu$ in $U_{3}$, by pulling back the standard Beltrami coefficient $\mu_{0}=0$ under $\phi_{1}$, that is $\mu=\phi_{1}^{*}\left(\mu_{0}\right)$ on $U_{3}$ or equivalently, $\mu=\bar{\partial} \phi_{1} / \partial \phi_{1}$. Observe that $\mu$ is invariant by $\left.F\right|_{U_{3}}$ by the construction, and it has bounded dilatation because $\phi_{1}$ is quasiconformal.

We can extend $\mu$ to the backward orbit of $U_{3}$ by letting $\mu:=\left(F^{n}\right)^{*}(\mu)$ on $F^{-n}\left(U_{3}\right)$, for every $n \geq 1$. Finally we can extend it to all of $\widehat{\mathbb{C}}$ by letting $\mu=\mu_{0}$ outside $\bigcup_{n=0}^{\infty} F^{-n}\left(U_{3}\right)$.

Since $\mu$ on $U_{3}$ is invariant by $\left.F\right|_{U_{3}}$, the extended $\mu$ is invariant by $F$. Also, since $F$ is analytic outside $\bar{U}_{2}$ it follows that $\|\mu\|_{\infty}=\left\|\mu_{U_{3}}\right\|_{\infty}<1$. Hence, $F$ is holomorphic with respect to the almost complex structure defined by $\mu$, and therefore we can apply the Measurable Riemann Mapping theorem (see e.g. [BF14, Theorem 1.27]) to obtain a quasiconformal homeomorphism $\Psi: \widehat{\mathbb{C}} \rightarrow \widehat{\mathbb{C}}$, satisfying $\mu=\Psi^{*}\left(\mu_{0}\right)$ or, equivalently, $\bar{\partial} \Psi=\mu \partial \Psi$. Since $\Psi$ is unique up to fixing the image of three points, we can normalize it by requiring $\Psi\left(\omega_{2}\right)=\omega=-e^{2 \pi i \theta} / 2, \Psi(0)=0$, and $\Psi(\infty)=\infty$.

The map $Q=\Psi \circ F \circ \Psi^{-1}$ is a quadratic polynomial. Indeed, it is a rational map of degree two with $Q^{-1}(\infty)=\infty$. Observe that 0 is fixed by $Q$. Since $\phi_{1}$ is conformal on $U_{1}$, so is $F$, and $F^{\prime}(0)=R_{\theta}^{\prime}(0)=e^{2 \pi i \theta}$. This implies that $\Psi$ is also conformal on $U_{1}$ and hence $Q^{\prime}(0)=F^{\prime}(0)=e^{2 \pi i \theta}$. Hence $z=0$ is a Siegel point of $Q$. Additionally, $\omega$ is a critical point, thus we conclude that $Q=P$.

Let $\Phi:=\Psi^{-1}$ and $D:=\Psi\left(U_{2}\right)$ (see Figure 3). We now show that $\Phi$ satisfies properties (a) to (d) of Theorem 1.1.

Property (a). First notice that $\phi_{1} \circ \Phi$ is conformal on $\Psi\left(U_{3}\right)$ and conjugates $P$ to $R_{\theta}$ on this domain. So $\Psi\left(U_{3}\right) \subset S$. By maximality of $H, \Psi\left(U_{3}\right)=S$, because if $\Psi\left(U_{3}\right)$ were only a subdisk of $S$ then $H$ would not the maximal domain of linearization. By construction, $\Phi$ conjugates $P$ to $F$ everywhere, but since $F=f$ except on $U_{2}, \Phi$ conjugates $P$ to $f$ everywhere except on $D$. Finally, since $U_{2}$ is compactly contained in $U_{3}$, we know $D$ is compactly contained in $S$.

Property (b). We have $\partial A_{P}(\infty)=J(P)$. On $A_{P}(\infty), \Phi$ conjugates $P$ to $f$. Hence 
$\Phi\left(A_{P}(\infty)\right) \subset A_{f}(\infty)$. Similarly $\Psi$ conjugates $f$ to $P$ on $A_{f}(\infty)$, and therefore $A_{f}(\infty) \subset$ $\Phi\left(A_{P}(\infty)\right)$. Hence $\Phi\left(A_{P}(\infty)\right)=A_{f}(\infty)$, and $\Phi(J(P))=\partial \Phi\left(A_{P}(\infty)\right)=\partial A_{f}(\infty)$. We have already seen that $S=\Psi\left(U_{3}\right)$. Hence $\Phi(\partial S)=\partial U_{3}=\partial_{2} H$. That $\Phi(\omega)=\omega_{2}$ is evident from the normalization of $\Psi$.

Property (c). By the construction, $\bar{\partial} \Psi=0$ a.e. on the complement of $\widehat{U}=\bigcup_{n=0}^{\infty} F^{-n}\left(U_{2}\right)$. Let $E \subset \mathbb{C} \backslash \widehat{U}$ be the measure zero set where $\Psi$ is not differentiable (in the real sense) or it is differentiable but either $|\partial \Psi|=|\bar{\partial} \Psi|$ or $\bar{\partial} \Psi \neq 0$. Obviously $\bar{\partial} \Phi=0$ on the complement of $\mathcal{D} \cup \Psi(E)=\Psi(\widehat{U}) \cup \Psi(E)$. But since $\Psi$ is quasiconformal and quasiconformal maps are absolutely continuous with respect to Lebesgue measure, $\Psi(E)$ has measure zero. We conclude that $\bar{\partial} \Phi=0$ almost everywhere on the complement of $\mathcal{D}$.

Property (d). In view of Theorem 2.4, it is enough to prove that $\omega$ is a measurable deep point of the complement of $\mathcal{D}$. To show this, McMullen has done the heavy lifting by proving Theorem 2.3: $\omega$ is a measurable deep point of $S_{\epsilon}$. Since $D$ is a definite distance away from $\omega$ and again using the $J(P)$ has measure zero, we deduce that $\omega$ is a measurable deep point of $S_{\epsilon} \backslash(D \cup J(f))$. Hence it is enough to show that $S_{\epsilon} \backslash(D \cup J(f)) \subset \mathbb{C} \backslash \mathcal{D}$.

The preimage $P^{-1}(D)$ has two components: $D$ itself and another one $D^{\prime}$. If $z \in \mathcal{D} \backslash D$, some forward iterate of $z$ must hit $D^{\prime}$. Hence $z$ does not belong to $S_{\epsilon}$ if $\epsilon$ is less than the distance between $D$ and $D^{\prime}$. This proves the desired inclusion and concludes the proof of Theorem 1.1.

In the course of the proof, we showed that $\omega$ is a measurable deep point of $\mathbb{C} \backslash \mathcal{D}$. We shall use this later, so we formally state it.

Corollary 3.1. The critical point $\omega$ is a measurable deep point of $\mathbb{C} \backslash \mathcal{D}$.

\section{Preliminaries about $C^{1+\alpha}$-conformal mappings and tightly similar sets}

To prove the Main Theorem, we need to establish some elementary properties of $C^{1+\alpha_{-}}$ conformal homeomorphisms and tightly similar sets.

First we prove that $C^{1+\alpha}$-conformality extend to inverses.

Proposition 4.1. Let $\phi: U \rightarrow V$ be a homeomorphism between the open sets $U$ and $V$, and suppose $\phi$ is $C^{1+\alpha}$-conformal at $z_{0} \in U$ with $\phi^{\prime}\left(z_{0}\right) \neq 0$. Then $\phi^{-1}$ is $C^{1+\alpha}$-conformal at $\phi\left(z_{0}\right)$.

Proof. We can assume that $z_{0}=\phi\left(z_{0}\right)=0$ and $\phi^{\prime}(0)=1$, so by the hypothesis

$$
\phi(z)=z+R(z), \text { where }|R(z)|<M|z|^{1+\alpha} \text { when }|z|<\delta
$$

for some $M$ and $\delta>0$. When $|z|$ is small enough, $|\phi(z)| \geq|z| / 2$. Hence there exists $\delta^{\prime}>0$ so that when $w \in B\left(0, \delta^{\prime}\right),\left|\phi^{-1}(w)\right| \leq 2|w|$ and $\phi^{-1}(w) \in B(0, \delta)$. Since $w=\phi\left(\phi^{-1}(w)\right)$ we can write

$$
w=\phi^{-1}(w)+R\left(\phi^{-1}(w)\right)
$$

and hence we have

$$
\phi^{-1}(w)=w-R\left(\phi^{-1}(w)\right)=w+\tilde{R}(w)
$$

with

$$
|\tilde{R}(w)|=\left|R\left(\phi^{-1}(w)\right)\right| \leq M\left|\phi^{-1}(w)\right|^{1+\alpha} \leq M|2 w|^{1+\alpha}
$$

when $w \in B\left(0, \delta^{\prime}\right)$, which concludes the proof.

The following Proposition is straightforward and left as an exercise for the reader.

Proposition 4.2. Let $A \subset B \subset C$ be compact sets. Then $A \underset{z_{0}}{\sim} C$ implies $A \underset{z_{0}}{\sim} B \underset{z_{0}}{\sim} C$. 
We end this section by showing that tight similarity at a point is preserved by homeomorphisms which are $C^{1+\alpha}$-conformal at that point.

Proposition 4.3. Let $A$ be a compact set and $\phi$ be a homeomorphism which is $C^{1+\alpha}$ conformal at $z_{0}$.

1. If $\phi\left(z_{0}\right)=z_{0}, \phi^{\prime}\left(z_{0}\right)=1$ then $A \underset{z_{0}}{\sim} \phi(A)$.

2. If $\phi^{\prime}\left(z_{0}\right) \neq 0$ and $B$ is a compact such that $A \underset{z_{0}}{\sim} B$, then $\phi(A) \underset{\phi\left(z_{0}\right)}{\sim} \phi(B)$.

Proof. To see the first statement, first write $\phi(z)=z+R(z)$, where $|R(z)| \leq M \mid z-$ $\left.z_{0}\right|^{1+\alpha}$ for $|z|$ small. Then, for any $a \in A \cap B\left(z_{0}, \delta\right)$,

$$
|a-\phi(a)| \leq M\left|a-z_{0}\right|^{1+\alpha} \text {. }
$$

This is the first requirement of Definition 1.3. The second requirement follows by applying the same argument to $\phi^{-1}$, which is $C^{1+\alpha}$-conformal by Proposition 4.1.

We can deduce the second statement from the first. Since simultaneously scaling $A$ and $B$ around $z_{0}$ by the same factor keeps them tightly similar, we can suppose $\phi^{\prime}\left(z_{0}\right)=1$. Then

$$
\phi(A) \underset{z_{0}}{\sim} A \underset{z_{0}}{\sim} B \underset{z_{0}}{\sim} \phi(B),
$$

so the two image sets are tightly similar.

Remark 4.4. From this proposition, together with Theorem 2.6 , we can see that $S$ is tightly self similar if $\theta$ is a quadratic irrational. Indeed, let $s$ be the eventual period of the continued fraction expansion of $\theta$, and first assume it is even. Let $\psi$ be the homeomorphism and $\kappa$ the scaling factor in Theorem 2.6, which ensure self-similarity of $S$. Then, the map

$$
h(z)=(1 / \kappa)(\psi(z+\omega)-\omega)
$$

sends $\kappa(\partial S-\omega)$ to $\partial S-\omega$, and is of the form $h(z)=z+O\left(|z|^{1+\alpha}\right)$. It follows from Proposition 4.3 that $\partial S-\omega \widetilde{\sim} \kappa(\partial S-\omega)$. When $s$ is odd, $h(z)=(1 / \kappa)(\overline{\psi(z+\omega)-\omega})$ gives the tight self-similarity between $\partial S-\omega$ and $\kappa(\overline{\partial S-\omega})$.

\section{Geometry invariance. Proof of the Main Theorem and Corollary 1.4}

In this section we prove the Main Theorem. Most of the statements are fairly straightforward by the results we have established so far. The delicate part is to prove that $\partial A_{f}(\infty) \underset{\omega_{2}}{\sim} J(f)$.

Recall that $\Phi$ denotes the quasiconformal homeomorphism given in Theorem 1.1, conjugating $P$ to $f$ on $\widehat{\mathbb{C}} \backslash D$, which is conformal outside $\mathcal{D}=\bigcup_{n=0}^{\infty} P^{-n}(D)$. Our strategy will be to prove that $J(P) \underset{\omega}{\sim} \overline{\mathcal{D}}$. Since $\Phi$ is $C^{1+\alpha}$-conformal at $\omega, \Phi(J(P))=\partial A_{f}(\infty)$, and we shall see that $\Phi(\overline{\mathcal{D}})$ contains $J(f)$, this will be sufficient to prove the desired similarity by Propositions 4.2 and 4.3 .

The idea in proving $J(P) \underset{\omega}{\sim} \overline{\mathcal{D}}$ is that the area of components of $\mathcal{D}$ must quickly decrease as we approach $\omega$. By bounded geometry, this means that the diameter of the components of $\mathcal{D}$ must also quickly decrease. Each connected component $D^{\prime}$ of $\mathcal{D}$ is contained in a Fatou component $F^{\prime}$. The modulus of the annulus $F^{\prime} \backslash D^{\prime}$ is independent of which component $D^{\prime}$ we are considering and so for any $z \in D^{\prime}$ the distance $d\left(z, \partial F^{\prime}\right)$ is comparable to the diameter of $D^{\prime}$, i.e., quickly decreasing as we approach $\omega$. 
Definition 5.1. Let $U$ be a bounded simply connected domain and $z \in U$. The inner radius $\underline{\mathrm{r}}(U, z)$ is given by

$$
\sup \{r: B(z, r) \subset U\}
$$

and the outer radius $\overline{\mathrm{r}}(U, z)$ is

$$
\inf \{r: U \subset B(z, r)\} .
$$

Claim 1. There exists $K_{1} \in(1, \infty)$ such that for any connected component $D^{\prime}$ of $\mathcal{D}$,

$$
1 \leq \frac{\overline{\mathrm{r}}\left(D^{\prime}, \alpha^{\prime}\right)}{\underline{\mathrm{r}}\left(D^{\prime}, \alpha^{\prime}\right)} \leq K_{1}
$$

where $\alpha^{\prime}$ denotes the the "center" of $D^{\prime}$, i.e., the unique point in $D^{\prime}$ whose forward orbit eventually hits the fixed point 0 .

The claim follows from Koebe's Distortion Theorem (see e.g. [CG93]) . Let $\phi: S \rightarrow$ $B(0,1)$ denote a map linearizing $P$, and $F^{\prime}$ be the Fatou component that contains $D^{\prime}$. When $n$ is sufficiently large, $\phi \circ P^{n}: F^{\prime} \rightarrow B(0,1)$ is a conformal isomorphism which, by the construction of $D$ (see Section 3) maps $D^{\prime}$ onto a round disk $B(0, s)$, and $\alpha^{\prime}$ to the origin (note that $s$ depends only on $D$ and not on the choice of the component $D^{\prime}$ ). Applying Koebe's Distortion Theorem to the inverse map, we get

$$
\frac{\overline{\mathrm{r}}\left(D^{\prime}, \alpha^{\prime}\right)}{\underline{\mathrm{r}}\left(D^{\prime}, \alpha^{\prime}\right)} \leq\left(\frac{1+s}{1-s}\right)^{2}
$$

Claim 2. There exists $K_{2}, K_{3} \in(0, \infty)$ such that for any connected component $D^{\prime}$ of $\mathcal{D}$,

$$
K_{2} \sqrt{\text { Area } D^{\prime}} \leq \overline{\mathrm{r}}\left(D^{\prime}, \alpha^{\prime}\right) \leq K_{3} \sqrt{\text { Area } D^{\prime}}
$$

where $\alpha^{\prime}$ is the center of $D^{\prime}$.

The claim is trivial, since

$$
\pi\left(\underline{r}\left(D^{\prime}, \alpha^{\prime}\right)\right)^{2} \leq \text { Area } D^{\prime} \leq \pi\left(\bar{r}\left(D^{\prime}, \alpha^{\prime}\right)\right)^{2}
$$

and the inner and outer radii are comparable by Claim 1 .

Claim 3. There exists $K_{4} \in(2, \infty)$ such that for any connected component $D^{\prime}$ of $\mathcal{D}$, and any $z \in D^{\prime}$,

$$
d(z, J(P))<K_{4} \overline{\mathrm{r}}\left(D^{\prime}, \alpha^{\prime}\right),
$$

where $\alpha^{\prime}$ is the center of $D^{\prime}$.

This claim is a consequence of Gröztsch's inequality. Letting $F^{\prime}$ be the component of the Fatou set that contains $D^{\prime}$, the modulus of $F^{\prime} \backslash \bar{D}^{\prime}$ is equal to the modulus $m$ of $S \backslash \bar{D}$. Put $\overline{\mathrm{r}}=\overline{\mathrm{r}}\left(D^{\prime}, \alpha^{\prime}\right)$. There exists a constant $c>1$ only depending on $m$, such that the modulus of $B\left(\alpha^{\prime}, c \overline{\mathrm{r}}\right) \backslash \bar{B}\left(\alpha^{\prime}, \overline{\mathrm{r}}\right)$ equals $2 m$ (we could have chosen any other number larger than $m$ ). If $B\left(\alpha^{\prime}, c \overline{\mathrm{r}}\right)$ were contained in $F^{\prime}$, then $B\left(\alpha^{\prime}, c \overline{\mathrm{r}}\right) \backslash \bar{B}\left(\alpha^{\prime}, \overline{\mathrm{r}}\right)$ would be contained in $F^{\prime} \backslash \bar{D}^{\prime}$, contradicting Gröztsch's inequality. So there exists $z^{\prime} \in \partial F^{\prime} \subset J(P)$ with $\left|z^{\prime}-\alpha^{\prime}\right| \leq c \overline{\mathrm{r}}$. By the triangle inequality, $\left|z-z^{\prime}\right| \leq\left|z-\alpha^{\prime}\right|+\left|\alpha^{\prime}-z^{\prime}\right| \leq \overline{\mathrm{r}}+c \overline{\mathrm{r}}$, which proves the claim, with $K_{4}:=c+1$.

Remark: By applying Koebe's distortion theorem to the the map $g:=\left(\phi \circ P^{n}\right)^{-1}$ : $B(0,1) \rightarrow F^{\prime}$, one can actually show that both quantities in Claim 3 are comparable. More precisely, setting $w:=g^{-1}(z)$,

$$
d(z, J(P))=d\left(z, \partial F^{\prime}\right) \asymp\left(1-|w|^{2}\right)\left|g^{\prime}(w)\right| \asymp\left|g^{\prime}(0)\right| \asymp \overline{\mathrm{r}}\left(D^{\prime}, \alpha^{\prime}\right) .
$$

Corollary 3.1 tells us that $\omega$ is measurable deep of the complement of $\mathcal{D}$, i.e. there exist constants $\beta, \delta, M>0$ so $\operatorname{Area}(\mathcal{D} \cap B(\omega, r)) \leq M r^{2+\beta}$, whenever $r<\delta$. 
Claim 4. There exists $\delta^{\prime}, \beta, K>0$, so that

$$
d(z, J(P)) \leq K|z-\omega|^{1+\beta / 2}
$$

whenever $z \in \mathcal{D} \cap B\left(\omega, \delta^{\prime}\right)$.

Fix $r_{0}>0$ small enough so that

- $0<r_{0}<\frac{\delta}{4}$, and

- $4\left(K_{3} \sqrt{M}\right)^{\gamma} r \leq r^{\gamma}$ whenever $0<r \leq r_{0}$. Here $\gamma:=(1+\beta / 2)^{-1}<1$.

There are only finitely many components $D^{\prime}$ of $\mathcal{D}$ with $\overline{\mathrm{r}}\left(D^{\prime}, \alpha^{\prime}\right)>r_{0}$. If there were infinitely many, then by Claim 2, the area of $\mathcal{D}$ would be infinite, but $\mathcal{D}$ is contained in the filled-in Julia set of $P$ and thus has finite area. Hence we can pick $\delta^{\prime}>0$ so that

- $\delta^{\prime} \leq \delta / 2$

- $B\left(\omega, \delta^{\prime}\right)$ does not meet a component of $\mathcal{D}$ with $\overline{\mathrm{r}}\left(D^{\prime}, \alpha^{\prime}\right)>r_{0}$.

Consider an arbitrary $z \in \mathcal{D} \cap B\left(\omega, \delta^{\prime}\right)$. Such $z$ lies in a component $D^{\prime}$ of $\mathcal{D}$, and we have chosen $\delta^{\prime}$ so that $r:=\overline{\mathrm{r}}\left(D^{\prime}, \alpha^{\prime}\right) \leq r_{0}$. Notice $D^{\prime} \subset B(\omega,|z-\omega|+2 r)$. As $|z-\omega|+2 r<\delta^{\prime}+2 r_{0}<\delta$, we obtain

$$
\text { Area } D^{\prime} \leq \text { Area } \mathcal{D} \cap B(\omega,|z-\omega|+2 r) \leq M(|z-\omega|+2 r)^{2+\beta} .
$$

Using Claim 2, we get

$$
r \leq K_{3} \sqrt{M}(|z-\omega|+2 r)^{1+\beta / 2}
$$

so

$$
r^{\gamma}-2 r\left(K_{3} \sqrt{M}\right)^{\gamma} \leq\left(K_{3} \sqrt{M}\right)^{\gamma}|z-\omega| .
$$

Since $r \leq r_{0}$, and by the choice of $r_{0}$, the left hand side is bounded below by $\frac{r^{\gamma}}{2}$, and we obtain

$$
r \leq K_{5}|z-\omega|^{1+\beta / 2}
$$

for some $K_{5} \in(0, \infty)$. By Claim $3, d(z, J(P)) \leq K_{4} K_{5}|z-\omega|^{1+\beta / 2}$ which proves Claim 4.

Claim 5. $J(P) \underset{\omega}{\sim} \overline{\mathcal{D}}$.

We have just established one of the two requirements of Definition 1.3. The set $\overline{\mathcal{D}}$ is a completely invariant closed set containing more than two points, so $J(P) \subset \overline{\mathcal{D}}$. Hence, the other requirement is automatically satisfied.

Proof of the Main Theorem. By Theorem 1.1, $\Phi(J(P))=\partial A_{f}(\infty)$. Again using Theorem 1.1 we obtain that $L:=\Phi^{\prime}(\omega) \neq 0$. Let $h(z):=z / L+\omega$, and $g(z):=z-\omega_{2}$. Then, the map $\Psi:=g \circ \Phi \circ h$ maps $L(J(P)-\omega)$ onto $\partial A_{f}(\infty)-\omega_{2}$. Moreover $\Psi$ maps the origin to itself, is $C^{1+\alpha}$-conformal and has derivative 1 at this point. By Proposition 4.3 ,

$$
L(J(P)-\omega) \underset{0}{\sim} \partial A_{f}(\infty)-\omega_{2} .
$$

Let us now see that $\partial A_{f}(\infty) \subset J(f) \subset \Phi(\overline{\mathcal{D}})$. The first inclusion is immediate. If $z \in J(f) \backslash \Phi(\mathcal{D})$, then $u:=\Phi^{-1}(z)$ has a bounded forward orbit that avoids $D$. Since the orbit of $u$ cannot hit $S \backslash D$ (otherwise the orbit of $z$ would hit $H$ ), we must have $\Phi^{-1}(z) \in J(P) \subset \overline{\mathcal{D}}$. Hence $z \in \Phi(\overline{\mathcal{D}})$.

By claim 5 and Proposition 4.3, $A_{f}(\infty) \underset{\omega_{2}}{\sim} \Phi(\overline{\mathcal{D}})$, Proposition 4.2 shows that $\partial A_{f}(\infty) \underset{\omega_{2}}{\sim}$ $J(f)$. This finishes the proof of part (a). 
To see part (b), notice that $\partial S$ is self-similar by Remark 4.4. The mapping $\psi$ of Theorem 2.6 can be turned into mapping of $\partial_{2} H$ by letting $\tilde{\psi}=\Phi \circ \psi \circ \Phi^{-1}$. By Propostion 4.1 this composition is $C^{1+\alpha}$-conformal or anticonformal, and has the same conformal or anticonformal derivative $\kappa$ as $\psi$. We can conclude that $\partial_{2} H$ admits a map such as the one in Theorem 2.6, and by Remark 4.4 is tightly self-similar.

For part (c), assume that $S$ contains an open triangle $T$ with vertices $a, b$ and $\omega$. Shrinking $T$ we can assume $T \cap \partial S=\{\omega\}$. We know from Theorem 1.1 (d) that $\Phi$ is $\mathbb{C}$-differentiable at $\omega$ and $\Phi^{\prime}(\omega) \neq 0$. Moreover, by Proposition 4.1 , we have that $\Phi^{-1}$ is also $\mathbb{C}$-differentiable at $\omega_{2}$. The image $\Phi([\omega, a])$ is a curve in $H$ having a tangent at the starting point, and similarly for $\Phi([\omega, b])$. The angle between the two tangents is the same as the angle of $T$ at $\omega$, since $\Phi^{\prime}(\omega)$ exists and is non-vanishing. Thus a line segment in the gap between the two tangents will not intersect the two image curves in a small enough neighborhood of $\omega_{2}$. Hence there is room for a triangle in $H$ with a vertex at $\omega_{2}$. As the inverse map $\Phi^{-1}$ is $\mathbb{C}$-differentiable at $\omega_{2}$ with non-vanishing derivative, we can use the exact same argument to establish that the existence of a triangle in $H$ with a vertex at $\omega_{2}$ implies the existence of a triangle in $S$ with a vertex at $\omega$.

Remark 5.2. The proof shows that $\partial_{2} H$ admits a map such as the one in Theorem 2.6, which is stronger than part (b).

It only remains to prove Corollary 1.4. The statements of Theorem 1.1 and the Main Theorem hold true if we replace $\omega_{2}$ with $\omega_{1}$. When $f$ is viewed as a mapping of the sphere, $\omega_{1}$ and $\omega_{2}$ do not play different roles. If we change coordinates by the map $z \mapsto 1 / z$, then $f$ takes the form $g(z)=1 / f(1 / z)=b^{-1} z^{2} \frac{a z+1}{z+a}$, i.e. we get a mapping of the form covered by Theorem 1.1 and the Main Theorem. The change of coordinates interchanges outer and inner boundary components of $H$ and maps $\omega_{1}$ for $f$ to $\omega_{2}$ for $g$, and $\omega_{2}$ for $f$ to $\omega_{1}$ for $g$. In particular, in proving the corollary, we can suppose $f^{m}(u)=\omega_{2}$.

Let $k \geq 1$ and $v$ be such that $P^{k}(v)=\omega$. There exists an inverse branch of $P$ mapping a neighborhood of $\omega$ conformally onto a neighborhood of $v$. Indeed, the boundary of $S$ is contained in the accumulation set of the forward orbit of $\omega$ which must be infinite. So $P^{j}(v) \neq \omega$, for $j=0,1, \ldots, k-1$. Since $\omega$ is the only zero of $P^{\prime}$, this implies in particular that $\left(P^{k}\right)^{\prime}(v) \neq 0$, which guarantees the existence of the local inverse branch.

The same argument shows that if $k \geq 1$ and $f^{k}(u)=\omega_{2}$, then there exists an inverse branch of $f^{k}$ mapping a neighborhood of $\omega_{2}$ onto a neighborhood of $u$.

Proof of Corollary 1.4. Part (a). We know that $\Phi$ conjugates $P$ to $f$ on $\widehat{\mathbb{C}} \backslash D$. Hence $\Phi$ conjugates $P^{n}$ to $f^{n}$ on $\widehat{\mathbb{C}} \backslash \bigcup_{j=0}^{n-1} P^{-j}(D)$. That means $\Phi=f^{-n} \circ \Phi \circ P^{n}$ in a neighborhood of $\omega^{\prime}$, for a suitably chosen branch of $f^{-n}$. Since the property of being $C^{1+\alpha}$ at a point is clearly preserved under composition with conformal mappings, we conclude that $\Phi$ is $C^{1+\alpha}$-conformal at $\omega^{\prime}$.

Part (b). Using a suitably chosen branch of $P^{-n}$ and Proposition 4.3, we get

$$
L_{1}(J(P)-\omega) \underset{0}{\sim} J(P)-\omega^{\prime},
$$

with $L_{1}=\left(P^{n}\right)^{\prime}\left(\omega^{\prime}\right)$. Using a suitably chosen branch of $f^{-m}$ in the same way, we get

$$
J(f)-\omega_{2} \underset{0}{\sim} L_{2}(J(f)-u)
$$

with $L_{2}=\left(f^{m}\right)^{\prime}(u)$. It follows from the Main Theorem that (b) holds with $L^{\prime}=$ $L_{1} \Phi^{\prime}(\omega) L_{2}^{-1}$.

Part (c). There exists an inverse branch $f^{-m}$ that maps $\omega_{2}$ to $u$, and maps the part of the boundary of $H$ that lies in some neighborhood of $\omega_{2}$ onto the part of the boundary of $H^{\prime}$ that lies in some neighborhood of $u$. If $\phi$ is the map expressing the self-similarity at $\omega_{2}$, then $f^{-n} \circ \phi \circ f^{n}$ will be a map that expresses self similarity of $\partial H^{\prime}$ at $u$. 


\section{References}

[BF14] Bodil Branner and Núria Fagella, Quasiconformal surgery in holomorphic dynamics, Cambridge studies in advanced mathematics, no. 141, Cambridge University Press, 2014.

[BFGH05] Xavier Buff, Núria Fagella, Lukas Geyer, and Christian Henriksen, Herman rings and Arnold disks, J. London Math. Soc. (2) 72 (2005), no. 3, 689-716. MR 2190332 (2007b:37094)

[BH99] Xavier Buff and Christian Henriksen, Scaling ratios and triangles in Siegel disks, Math. Res. Lett. 6 (1999), no. 3-4, 293-305. MR 1713131 (2000h:37069)

[Brj65] A. D. Brjuno, On convergence of transforms of differential equations to the normal form, Dokl. Akad. Nauk SSSR 165 (1965), 987-989. MR 0192098 (33 \#325)

[CG93] Lennart Carleson and Theodore W. Gamelin, Complex dynamics, Universitext: Tracts in Mathematics, Springer-Verlag, New York, 1993. MR 1230383 (94h:30033)

[DH85] Adrien Douady and John Hamal Hubbard, On the dynamics of polynomiallike mappings, Ann. Sci. École Norm. Sup. (4) 18 (1985), no. 2, 287-343. MR 816367 (87f:58083)

[Fat19] P. Fatou, Sur les équations fonctionnelles, Bull. Soc. Math. France 47 (1919), 161-271.

[Khi97] A. Ya. Khinchin, Continued fractions, russian ed., Dover Publications, Inc., Mineola, NY, 1997, With a preface by B. V. Gnedenko, Reprint of the 1964 translation. MR 1451873 (98c:11008)

[LV73] O. Lehto and K. I. Virtanen, Quasiconformal mappings in the plane, second ed., Springer-Verlag, New York-Heidelberg, 1973, Translated from the German by K. W. Lucas, Die Grundlehren der mathematischen Wissenschaften, Band 126. MR 0344463 (49 \#9202)

[McM96] Curtis T. McMullen, Renormalization and 3-manifolds which fiber over the circle, Annals of Mathematics Studies, vol. 142, Princeton University Press, Princeton, NJ, 1996. MR 1401347 (97f:57022)

[McM98]_, Self-similarity of Siegel disks and Hausdorff dimension of Julia sets, Acta Math. 180 (1998), no. 2, 247-292. MR 1638776 (99f:58172)

[Mil06] John Milnor, Dynamics in one complex variable, third ed., Annals of Mathematics Studies, vol. 160, Princeton University Press, Princeton, NJ, 2006. MR 2193309 (2006g:37070)

[Pet96] Carsten Lunde Petersen, Local connectivity of some Julia sets containing a circle with an irrational rotation, Acta Math. 177 (1996), no. 2, 163-224. MR 1440932 (98h:58164)

[Rüs67] Helmut Rüssmann, Über die Iteration analytischer Funktionen, J. Math. Mech. 17 (1967), 523-532. MR 0218535 (36 \#1621)

[Shi86] Mitsuhiro Shishikura, Surgery of complex analytic dynamical systems, Dynamical systems and nonlinear oscillations (Kyoto, 1985), World Sci. Adv. Ser. Dynam. Systems, vol. 1, World Sci. Publishing, Singapore, 1986, pp. 93-105. MR 854306 (87k:58135)

[Shi87] On the quasiconformal surgery of rational functions, Ann. Sci. École Norm. Sup. (4) 20 (1987), no. 1, 1-29.

[Sie42] Carl Ludwig Siegel, Iteration of analytic functions, Ann. of Math. (2) 43 (1942), 607-612. MR 0007044 (4,76c) 
[Sul85] Dennis Sullivan, Quasiconformal homeomorphisms and dynamics. I. Solution of the Fatou-Julia problem on wandering domains, Ann. of Math. (2) 122 (1985), no. 3, 401-418.

[Tan90] Lei Tan, Similarity between the Mandelbrot set and Julia sets, Comm. Math. Phys. 134 (1990), no. 3, 587-617. MR 1086745 (92k:58228)

[Yoc95] Jean-Christophe Yoccoz, Théorème de Siegel, nombres de Bruno et polynômes quadratiques, Astérisque (1995), no. 231, 3-88, Petits diviseurs en dimension 1. MR 1367353 (96m:58214)

[Zha11] Gaofei Zhang, All bounded type Siegel disks of rational maps are quasi-disks, Invent. Math. 185 (2011), no. 2, 421-466. MR 2819165 